INPLASY PROTOCOL

To cite: Tang et al. Effects of Mindfulness-based Intervention on Adolescents Emotional Disorders: a Systematic Review and Metaanalysis Protocol. Inplasy protocol 2021110054. doi: 10.37766/inplasy2021.11.0054

Received: 15 November 2021

Published: 15 November 2021

Corresponding author:

Fei Dai

df13437155783@163.com

Author Affiliation: Institute of Physical Education, Wuchang Institute of Technology.

Support: tem No.: 2020715.

Review Stage at time of this submission: Data analysis.

Conflicts of interest: None declared.

\section{Effects of Mindfulness-based Intervention on Adolescents Emotional Disorders: a Systematic Review and Meta-analysis Protocol}

Tang, DF1; Mo, LQ²; Zhou, XC³ Shu, JH4; Wu, L5; Wang, D6; Dai, $\mathrm{F}^{4}$.

Review question / Objective: Patientor population: (1) Adolescents (10-18 years old) with a diagnosis of mood disorder. (2) No limitation on duration of illness, gender or race. (3) No organic brain injury or other neuropsychiatric disorders. (4) No medications affecting the neurological or psychiatric system. (5) Have not participated in other clinical trials in the last 3 months. Experimental interventions: mindfulness practice, including yoga, tai chi, qigong, and meditation. Control interventions: Electrotherapy, medication or conventional treatment. Outcomes: We will use a metaanalysis of relevant randomized controlled trials to provide a rational and effective Mindfulness-based intervention program for alleviating mood disorders and psychological problems in adolescents.

INPLASY registration number: This protocol was registered with the International Platform of Registered Systematic Review and Meta-Analysis Protocols (INPLASY) on 15 November 2021 and was last updated on 15 November 2021 (registration number INPLASY2021110054).

\section{INTRODUCTION}

Review question / Objective: Patientor population: (1) Adolescents (10-18 years old) with a diagnosis of mood disorder. (2) No limitation on duration of illness, gender or race. (3) No organic brain injury or other neuropsychiatric disorders. (4) No medications affecting the neurological or psychiatric system. (5) Have not participated in other clinical trials in the last 3 months. Experimental interventions: mindfulness practice, including yoga, tai chi, qigong, and meditation. Control 
interventions: Electrotherapy, medication or conventional treatment. Outcomes: We will use a meta-analysis of relevant randomized controlled trials to provide a rational and effective Mindfulness-based intervention program for alleviating mood disorders and psychological problems in adolescents.

Condition being studied: The issue of adolescent mental health and emotional disorders has received increasing attention in recent years. Worldwide, $20 \%$ of adolescents (10-19 years of age), in any given year, experience a mental health problem, most commonly depression or anxiety. As a result, the World Health Organization's priorities for advancing the field of adolescent mental health in 2012 include improving adolescents' coping skills and adapting creative, complementary treatment models that can be implemented in low-resource settings. There is growing evidence that adolescents' inability to regulate their emotions due to psychological vulnerability, the presence of low selfesteem and emotional disorders are the main causes of various psychological disorders. Studies have reported a dramatic increase in the prevalence of emotional disorders in the adolescent population, and studies have reported emotional intelligence and internalization problems, depression and anxiety problems, and substance abuse problems that can be caused by emotional disorders, which will also become a major problem affecting adolescent development. Because of the many causes that trigger psychological disorders in adolescents, in addition to a range of therapeutic approaches such as biomedical interventions, electrotherapy and medication, some mindfulness practice such as yoga have gained widespread attention as interventions to improve mental health and deal with emotional disorders. Therefore, mindfulness practice have a positive impact on emotional disorders and psychological problems in adolescents and should be used as an adjunctive treatment to medication.

\section{METHODS}

Participant or population: (1) Adolescents (10-18 years old) with a diagnosis of mood disorder. (2) No limitation on duration of illness, gender or race. (3) No organic brain injury or other neuropsychiatric disorders. (4) No medications affecting the neurological or psychiatric system. (5) Have not participated in other clinical trials in the last 3 months.

Intervention: Mindfulness practice, including yoga, tai chi, qigong, and meditation.

Comparator: Electrotherapy, medication or conventional treatment.

Study designs to be included: Published randomized controlled trials(RCTs) of the effects of mindfulness practice on emotional disorders in adolescents reported in Chinese or English.

Eligibility criteria: We will only include randomized controlled trials (RCTs). Nonrandomized controlled trials, protocols, meetings, abstracts, incomplete texts, animal studies, systematic reviews, and meta-analyses were excluded.

Information sources: For literature on mindfulness practice for adolescent emotional disorders published before October 2021, search databases will include Google Scholar, EMBASE, Web of Science, PubMed, the CNKI, the Chinese Science and Technology Periodical Database, VIP, Wanfang, and Cochrane Library.

Main outcome(s): Changes in selfawareness, depression, and anxiety in adolescents.

Quality assessment / Risk of bias analysis: The two authors will evaluate the methodological quality of randomized controlled trials according to the Cochrane Handbook for the Evaluation of Intervention Systems (Higgins 2011), independently assessing the quality of trials included in the evaluation by hidden allocation 
(selection bias); blinding (performance bias and detection bias); participant blinding (performance bias); outcome assessment blinding (detection bias); completeness of outcome data ( attrition bias); selective reporting (reporting bias); and other biases. If any disagreements arise they will be resolved with a third investigator until consensus is reached. /If more than 10 studies are available for analysis, publication bias will be assessed by generating a funnel plot. A symmetrical distribution of data in the funnel plot indicates no publication bias. If it is not symmetrical, we will further analyze the reasons for bias and provide a reasonable explanation.

Strategy of data synthesis: Two authors independently extract the relevant information for each study. A third author will check all data for accuracy. Review Manager 5.3 (Cochrane Collaboration, Oxford, UK) will be used to assess risk of bias, heterogeneity, sensitivity and subgroup analysis. We will calculate weighted estimates across trials and interpret the results.

Subgroup analysis: We grouped gender, age, duration of illness, type of positive thinking practice, duration and frequency of practice to explore possible reasons for the high heterogeneity.

Sensitivity analysis: Sensitivity analysis will be performed by excluding tests one by one and observing whether there is a significant change in the synthesis results. If significant changes are present, further analysis will be performed and a prudent decision will be made whether to proceed with the synthesis. If there is no significant change, it indicates that the merger can be performed.

Country(ies) involved: China.

Keywords: Mindfulness,Adolescents, Emotional disorders, Systematic review, Meta-analysis.

Contributions of each author:

Author 1 - Danfeng Tang.
Email: tangdanfeng123123@163.com

Author 2 - Liqiong Mo.

Email: zhuzi1982_1982@163.com

Author 3 - Xinchu Zhou.

Email: zxinchu@163.com

Author 4 - Junhong Shu.

Email: sjh18627127865@163.com

Author 5 - Lei Wu.

Email: wulei1896@163.com

Author 6 - Dong Wang.

Email: wd18986023639@163.com

Author 7 - Fei Dai.

Email: df13437155783@163.com 